$p=0.011)$. And the peak level of AST (68.72 \pm 68.00 vs. $106.67 \pm 79.09$ $\mathrm{IU} / \mathrm{L}, p=0.008)$ and ALT (30.92 \pm 29.72 vs. $53.70 \pm 40.86 \mathrm{IU} / \mathrm{L}, p=0.002)$ were significant lower in the FOLE group compared with the FOLE group. However, the peak levels of serum triglyceride were similar. $(245.22 \pm 128.47$ vs. $226.97 \pm 99.49 \mathrm{mg} / \mathrm{dL}, p=0.747)$.

Conclusions The use of FOLE in VLBW infants may reduce the risk of PNALD.

\section{ADEQUATE POSTNATAL DIAGNOSTIC MODALITIES FOR PRENATALLY DIAGNOSED BILIARY CYSTIC MALFORMATIONS}

doi:10.1136/archdischild-2012-302724.0720

T Okada, S Honda, H Miyagi, A Taketomi. Department of Gastroenterological Surgery I, Hokkaido University Graduate School of Medicine, Sapporo, Japan

Background and Aims The aim of this study was to determine an appropriate postnatal diagnostic management plan for prenatally diagnosed biliary cystic malformation (BCM) patients.

Methods From 2002 to 2011, a total of 27 consecutive children with pancreaticobiliary maljunction (PBM) were treated at our institute. Eight (29.6\%) of our 27 patients with choledochal cyst (CC) were diagnosed prenatally and examined clinically. Prenatally diagnosed cystic biliary atresia (BA) was noted in 2 patients with type 1 cystic BA. The clinical data, preoperative imaging findings, and final diagnosis using intraoperative cholangiography were evaluated in these BCM patients.

Results Infants with prenatally diagnosed CC were divided into two groups after birth: a symptomatic group of 5 patients, and an asymptomatic group of 3 patients. According to CC patients, ultrasonography (US) did not reveal a PBM in all 8 CC patients, although the main pancreatic duct was shown in 2 CC patients. The PBM and main pancreatic duct were shown by MRCP at high rates of 80 and $60 \%$, respectively, compared with US and dynamic CT. In cystic BA patients, US and MRCP showed that the gallbladder was atrophic in both of the two cystic BA patients compared with the CC patients. There was not triangular cord sign in the two by US.

Conclusion This study clearly showed that, in some cases, such as prenatally diagnosed BCM, MRCP eliminates the need for endoscopic retrograde cholangiopancreatography (ERCP) because of its excellent sensitivity and specificity, thus avoiding an invasive procedure with marked radiation exposure.

\section{THE EFFECT OF THE RED WINE POLYPHENOL RESVERATROL ON CHOLESTASIS: ANTI-APOPTOTIC, MITOCHONDRIAL BIOGENESIS AND AUTOPHAGY}

doi:10.1136/archdischild-2012-302724.0721

'MM Tiao, ${ }^{2} \mathrm{~F}$ Huang, Mitochondrial Group. 'Pediatrics, Kaohsiung Chang Gung Memorial Hospital and Chang Gung University College of Medicine; 'Pediatrics, Kaohsiung Veterans General Hospital, Kaohsiung, Taiwan R.O.C.

Background and Aims Mitochondria are known to be involved in cholestatic liver injury. The potential protective effect of resveratrol in cholestatic liver injury and the possible roles of autophagy and apoptosis induction in this process are not yet clear. The aim of this study is to determine whether resveratrol administration after bile duct ligation can reduce cholestasis-induced liver injury through modulating apoptosis, mitochondrial biogenesis and autophagy.

Methods A rat model of cholestasis was established by bile duct ligation (BDL) and compared with a sham group receiving laparotomy without BDL, with resveratrol or control treatments following BDL. The expression of proteins involved in the apoptotic and autophagic pathways were analyzed by western blotting. Apoptosis was examined by TUNEL staining.

Results In the resveratrol/BDL group LC3-II upregulation persisted for 1-7 days, Bax was downregulated and catalase was upregulated at 3-7 days after resveratrol treatment. The decline in mitochondrial DNA copy number was reversed at 3-7 days. Caspase 3 expression was significantly downregulated at 3-7 days in the resveratrol group. TUNEL staining showed significant numbers of apoptotic liver cells appeared in livers 3-7 days after BDL and that was decreased by resveratrol treatment.

Conclusions Our results indicate that early resveratrol treatment reverses impaired liver function within hours of $\mathrm{BDL}$.

\section{HETEROGENEITY IN THE DIAGNOSIS OF COELIAC DISEASE IN PAEDIATRIC PATIENTS}

doi:10.1136/archdischild-2012-302724.0722

${ }^{1,2 \mathrm{D}}$ Croaker, ${ }^{3} \mathrm{~J}$ Swann, ${ }^{4} \mathrm{~L}$ Hallam, 1,2P Jenkins, ${ }^{5} \mathrm{C}$ Beardsley. ${ }^{1}$ Dept of Paediatrics and Child Health, The Canberra Hospital; '2Australian National University, Canberra, ACT, ${ }^{3}$ Wollongong University, Wollongong, NSW; ${ }^{4}$ Anatomical Pathology; ${ }^{5}$ Department of Surgery, The Canberra Hospital, Canberra, ACT, Australia

Background "Gluten intolerance" is commonly diagnosed and often confused in the public mind with coeliac disease. Authors in Western Australia recently demonstrated an approximately 5\% rate of coexistence of eosinophilic oesophagitis (EO) with villous atrophy. We asked whether our population was similar.

Methods We performed a retrospective chart review of all those with gastroscopy and small-bowel biopsy and a subsequent diagnosis of CD in children less than 16 years of age between 1 April 2003 and the 31st of June 2011.

Main results 239 gastroduodenoscopies were reviewed. Biopsy of both the oesophagus and duodenum was available in 231 patients. There were 124 patients positive for coeliac disease, 105 negative, and 10 indeterminate. 14 of 231 were positive for $\mathrm{EO}$, and 4 of the $126 \mathrm{CD}$ patients were also positive for $\mathrm{EO}$. Two of the four $\mathrm{CD}+\mathrm{EO}$ patients were rescoped during the time interval, and both were in remission for changes of $\mathrm{CD}$, although both still had changes of $\mathrm{EO}$ evident. There were $7 \mathrm{CD}$ patients reported with other forms of oesophageal inflammation.

Histologically of our four patients with EO and villous atrophy, at least three have potentially allergic changes instead of fullblown CD.

Conclusion There have been recent suggestions that serological and other tests may render the small bowel biopsy unnecessary in the management of $\mathrm{CD}$. We note that $\mathrm{EO}$ is associated with villous atrophy in 3 to $4 \%$ cases. Serology and symptomatology presenting as coeliac disease continues to warrant detailed investigation, including endoscopic work up.

\section{REVIEW OF TTG VERSUS SMALL INTESTINAL BIOPSY RESULTS; DO WE STILL NEED DUODENAL BIOPSY TO DIAGNOSE COELIAC DISEASE?}

doi:10.1136/archdischild-2012-302724.0723

TIY Hassan, S Quinn. Paediatrics, AMNCH, Dublin, Ireland

Aims To assess compare the results of tissue transglutaminase (tTg) with small intestinal biopsy results in children who had oesphageogastroduodenoscopy (OGD) to assess for coeliac disease (CD) at the National Children's Hospital, Tallaght, between January 2008 and December 2009.

Methods We reviewed the patients' records for all OGDs performed to assess for CD during the study period. Small intestinal biopsy results versus the $\mathrm{t} \mathrm{T}$ results were recorded.

Results 61 patients had an OGD performed during this period for assessment for CD (age 2-15 years). Three were excluded because no $\mathrm{tTg}$ was performed or recorded. Of these, 26 patients were males with male to female ratio of 0.8:1.

Fifty eight patients were included in the study, 32 had positive intestinal biopsy. 
Of these 32 patients 29 had positive tTg. This gives a sensitivity of $90 \%$. Thirteen patients had tTg greater than 100,12 had positive intestinal biopsy; this gives a sensitivity of $92 \%$.

Conclusion Although the sensitivity of thg in our series is $90 \%$, it is reasonable to assess small intestinal biopsy in before subjecting children to lifelong gluten free diet.

\section{HLA D02/D08 TYPING IN CHILDREN DIAGNOSED WITH CELIAC DISEASE}

doi:10.1136/archdischild-2012-302724.0724

G Lesanu, C Becheanu, M Stocklosa, I Tincu, R Smadeanu, R Vlad, D Pacurar. 'Grigore Alexandrescu' Emergency Children's Hospital, Bucharest, Romania

Background and Aims Genes encoding HLA DQ2/DQ8 are associated with celiac disease (CD) and testing for their presence has high negative predictive value for the diagnosis. The aim of this study was to assess the role of HLA typing in symptomatic individuals in whom the diagnosis of $\mathrm{CD}$ is uncertain.

Methods We proceded a retrospectiv study leaded on a group of children investigated for CD in "Grigore Alexandrescu" Emergency Children's Hospital from 2007 to 2012 that underwent HLA typing. Inclusion criteria were all patient with mild enteropathy (Marsh 1, 2, 3a), moderate elevated values of tisular tranglutaminase (tTG) antibodies (between cut off point and 5 times nomal value) and poor response to gluten free diet. The medical records of all patients investigated for $\mathrm{CD}$ were reviewed.

Results 164 patients were performed HLA typing; 26 patients satisfied the inclusion criteria; 20 (76.9\%) of these had HLA DQ2/DQ8 present and $6(23.07 \%)$ had a negative test for HLA DQ2/DQ8. The mean age of our investigated group was 23.46 months and the mean age for HLA DQ2/DQ8 negative group was 21.08 months. Sex distribution indicated 9 boys and 17 girls. Gastrointestinal symptoms dominated: 17 children had diarrhea, 9 had failure to thrive and 13 patients had both chronic diarrhea and poor weight gain.

Conclusion Patients with clinical suspicion of CD that have moderate levels of tTG antibodies, mild biopsy changes and poor response to gluten free diet need to have HLA typing specially at younger ages (under 3 years old).

\section{LYMPHOCYTE RESPIRATION IN CHILDREN WITH TRISOMY 21}

doi:10.1136/archdischild-2012-302724.0725

'E Aburawi, 'A Souid, ${ }^{2} \mathrm{H}$ Penefsky, 'T Pramathan. 'Pediatrics, United Arab Emirates University, FMHS, Al Ain, United Arab Emirates; ${ }^{2}$ Pediatrics, Ciricus, New York, NY, USA

\footnotetext{
Aims This study aimed to measure lymphocyte mitochondrial $\mathrm{O}_{2}$ consumption (cellular respiration) in children with trisomy 21.

Methods Peripheral blood mononuclear cells were isolated from whole blood of trisomy 21 and control children and immediately used to measure the respiratory rate. $\left[\mathrm{O}_{2}\right]$ was determined as function of time from the phosphorescence decay rates $(1 / \mathrm{t})$ of $\mathrm{Pd}$ (II)-meso-tetra-(4-sulfonatophenyl)-tetrabenzoporphyrin. In sealed vials containing cells and glucose as a respiratory substrate, $\left[\mathrm{O}_{2}\right]$ declined linearly with time, confirming the zero-order kinetics of $\mathrm{O}_{2}$ consumption (conversion to $\mathrm{H}_{2} \mathrm{O}$ ) by cytochrome oxidase.

Results The rate of respiration ( $\mathrm{k}$, in $\mathrm{mM} \mathrm{O}_{2}$ per min), thus, was the negative of the slope of $\left[\mathrm{O}_{2}\right]$ vs. time. $\mathrm{NaCN}$ inhibited $\mathrm{O}_{2}$ consumption, confirming the oxidation occurred in the mitochondrial respiratory chain. For control children (age $=8.8 \pm 5.6 \mathrm{yr}, \mathrm{n}=26$ ), the mean $\left( \pm \mathrm{SD}\right.$ ) value of $k_{c}$ (in $\mathrm{mM} \mathrm{O}_{2}$ per min per $10^{7}$ cells) was $1.36 \pm 0.79$ (coefficient of variation $=58 \%$; median $=1.17$; range $=0.60$ to $3.12 ;-2 \mathrm{SD}=0.61$ ). For children with trisomy 21 (age $=7.2 \pm 4.6 \mathrm{yr}$, $\mathrm{n}=26$ ), the value of $k$, was $0.82 \pm 0.62$ (coefficient of variation $=76 \%$; median $=0.60$; range $=0.20$ to 2.80$), p<0.001$. Fourteen of $26(54 \%)$ children with trisomy 21 had $k$, values of 0.20 to 0.60 (i.e., $<-2 S D$ ).
}

Conclusion Thus, it appears that some children with trisomy 21 have relatively reduced lymphocyte bioenergetics. The biological implication of this finding (variation) requires further studies.

\section{LERI-WEILL DYSCHONDROSTEOSIS - A CASE OF COMPLETE DELETION OF ONE OF THE COPIES OF SHOX GENE}

doi:10.1136/archdischild-2012-302724.0726

J Sales Marques, Al Pinto Pais, L Ferraz. Centro Hospitalar Vila Nova de Gaia/Espinho EPE, Vila Nova de Gaia, Portugal

Introduction Leri-Weill dyschondrosteosis (LWD), is a dominantly inherited skeletal dysplasia with disproportionate short stature owing to mesomelic shortening of the forearm and lower leg and Madelung deformity of the arm is found in $74 \%$ of children. SHOX mutations is found in $70 \%$ of cases.

Case Report Thirteen old month boy was admitted to genetic consultation because of short stature. The mother has disproportionate short stature. On physical examination, we found a phenotype similar with the mother, with short arms and lower legs. Height below the 5 th percentile. The skeletal x-ray confirmed mesomelic shortening of the forearm and lower legs. The x.ray did not demonstrated Madelung deformity of the arm. Molecular study using MLPA, confirmed complete deletion of one of the copies of SHOX gene - more than $440 \mathrm{~Kb}$. Later on, we confirmed that he has growth hormone deficiency. The mother has also LWD.

Discussion LWD should be included in the diagnoses of short stature, especially if the child is disproportionate and has family history. In our case, because the mother is affected, the deletion of the $\mathrm{SCHOX}$ gene is inherited in the pseudoautosomal region of $\mathrm{X}$ chromosome. The transmission is pseudodominant and so the daughters of the index case will inherited the $\mathrm{X}$ chromosome of the father and will be affected. The boys will inherited the Y chromosome of the father. Prenatal diagnosis and genetic counseling is available for this syndrome. Treatment options include administration of recombinant growth hormone to improve final adult height.

\section{WILSON'S DISEASE: A CHALLENGING DIAGNOSIS. CLINICAL MANIFESTATIONS AND DIAGNOSTIC PROCEDURES IN 32 PATIENTS}

doi:10.1136/archdischild-2012-302724.0727

\section{Mihai. Pediatric, Faculty of Medicine of Constanta, Constanta, Romania}

Introduction Wilson disease is a neurodegenerative disease of copper metabolism. The genetic defect, localized to chromosome arm 13q, has been shown to affect the copper-transporting adenosine triphosphatase (ATPase) gene (ATP7B) in the liver.

Material and Methods Our aim was to study the clinical and laboratory characteristics of 32 children and young adults diagnosed with WD and point out the diagnostic difficulties. The study was done between 2001 and 2011. Evaluation included detailed physical examination, conventional laboratory testing, genetic analysis, and liver biopsy.

Results Patients with hepatic symptoms showed a considerably earlier onset of symptoms and a shorter diagnostic delay before definitive diagnosis than those with neuropsychiatric symptoms. The mean age at diagnosis was $9.12+/-2.59$ years (range 5 years-20 years). 30 patients were symptomatic, 18 were referred because of abnormal liver function test results and/or hepatomegaly, 12 had neuropsychiatric symptoms and 2 received their diagnoses after family screening. Hepatic copper concentration was between 250 and $1200 \mathrm{microg} / \mathrm{g}$. 12 patients had liver cirrhosis, 16 chronic hepatitis, and 2 had massive hepatic necrosis on necropsy.

Conclusions Any person with recurrent hepatic disease and unexplained neurologic symptoms should be investigated to have 